\title{
Sorgende Gemeinschaften?
}

\author{
,Demenzfreundliche Kommunen' zwischen sozialstaatlichem Sparmodell und \\ Emanzipationsgewinn
}

\author{
Mike Laufenberg
}

\begin{abstract}
Der von Bund, Kommunen und Selbsthilfeorganisationen ausgerufene ,demenzfreundliche' Umbau der Gesellschaft wird einer kritischen Analyse unterzogen. Hierfür werden die polit-ökonomischen und wohlfahrtstaatlichen Rahmenbedingungen des sozialpolitischen Konzepts der, sorgenden Gemeinschaft' rekonstruiert, welches die Zivilgesellschaft zu einer wesentlichen Akteurin ,demenzsensibler' Kommunen erklärt. Unter den gegebenen Bedingungen läuft diese Entwicklung Gefahr, den mangelnden Ausbau sozialstaatlicher Transfer- und Dienstleistungen in der Altensorge abzufedern. Am Beispiel des Konzepts der , demenzfreundlichen Kommune' wird gezeigt, dass bürgerschaftliche Sorgepraktiken Menschen mit Demenz zugleich Emanzipationsgewinne in Form von steigender sozialer Partizipation und Selbstbestimmung ermöglichen. Ausgehend von diesem Spannungsverhältnis wird ein Vorschlag unterbreitet, wie gemeinwesenorientierte Care-Arrangements jenseits der Dichotomie von sozialstaatlichem Sparmodell und idealistisch verklärter Sorgegemeinschaft zu fassen wären.
\end{abstract}

Ersteinreichung: 24. Januar 2018; Veröffentlichung online: 20. Mai 2018

An english abstract can be found at the end of the document.

\section{Einleitung}

Demenz ist in den vergangenen zehn Jahren nicht nur zu einem zentralen Thema der Gesundheits- und Pflegepolitik, sondern auch zu einem sichtbaren Phänomen in deutschen Städten und Kommunen geworden[1]. In vielen Regionen und Städten sind Angehörigeninitiativen, ,demenzfreundliche Kommunen“ und ,Demenznetzwerke“ entstanden, die lokale Projekte und Angebote für Menschen mit Demenzdiagnose[2] anstoßen und vernetzen. Demenz wird dabei zu „einer großen sozialen und humanitären Herausforderung für das Gemeinwesen“ (Aktion Demenz e.V. o. J.) erklärt. Zwei Ziele stehen im Vordergrund: Erstens soll eine Verbesserung der bedarfsgerechten Versorgung und Unterstützung von Menschen mit kognitiven Veränderungen und ihrem sozialen Umfeld erreicht werden. Hierzu müssten kommunale Verwaltungen, professionelle Akteure aus Medizin und Pflege, zivilgesellschaftliche Organisationen und ehrenamtliche Helfer_innen enger miteinander kooperieren. Zweitens wird eine Entstigmatisierung sowie Verbesserung von sozialer Teilhabe und Inklusion von Menschen mit kognitiven Beeinträchtigungen angestrebt. Damit soll defizitorientierten 
Demenzbildern entgegengewirkt werden, die mit Viktimisierung und Marginalisierung einhergehen.

Der gegenwärtig von Bundes- bis Kommunalebene geforderte ,demenzfreundliche 'Umbau von Stadtgesellschaften wird im Folgenden einer kritischen Analyse unterzogen. Der Diskurs über Sorgeverhältnisse für Menschen mit Demenz wird hierbei sozialanalytisch als Verdichtungspunkt von heterogenen Strategien und Auseinandersetzungen verstanden, bei denen es um die Neujustierung des Verhältnisses von Individuum, (Wohlfahrts-)Staat und Gesellschaft geht. Um dies zu verdeutlichen, wird zunächst das neuere sozialpolitische Konzept der ,sorgenden Gemeinschaft' beleuchtet, welches die Zivilgesellschaft zu einer wesentlichen Akteurin des ,demenzfreundlichen“ Umbaus der Gesellschaft erklärt (Kapitel 1). Daran anschließend erfolgt eine knappe Rekonstruktion der polit-ökonomischen und wohlfahrtstaatlichen Rahmenbedingungen gegenwärtiger Bemühungen, Demenz als gesamtgesellschaftliche Herausforderung zu definieren (Kapitel 2). Kritiker_innen wenden hier ein, dass eine Definition urbaner Zivilgesellschaften als CareGemeinschaften den mangelnden Ausbau sozialstaatlicher Transfer- und Dienstleistungen abfedere sowie eine Ausbeutung des Ehrenamts und eine De-Professionalisierung von Pflegearbeit begünstige (Haubner 2017, van Dyk/Dowling/Haubner 2016).

Am konkreten Beispiel der bürgerrechtlichen Idee und Praxis von ,demenzfreundlichen Kommunen' zeigt die vorliegende Analyse auf, inwiefern ,sorgende Gemeinschaften 'jedoch komplexer und widersprüchlicher - und damit mehr als eine sozialstaatliche Entlastungsressource - sind (Kapitel 3). Um dies zu verdeutlichen, werden feministische und disability-theoretische Perspektiven auf citizenship und care herangezogen, die den Forderungen von Menschen mit Demenz nach umfassenden sozialen, politischen und zivilen Rechten Rechnung tragen. Bürgerschaftlich-kollektive Care-Settings, so das Argument, bergen für Menschen mit Demenz spezifische Emanzipationsgewinne in Form von steigender sozialer Partizipation und Selbstbestimmung. Ohne das Spannungsverhältnis zwischen sozialstaatlicher Aneignung und Emanzipationspotenzial gänzlich auflösen zu können, schließt der Beitrag mit einigen Überlegungen ab, wie aktuelle, im Zeichen von Altern und Demenz stehende, Transformationen von kommunalen Infrastrukturen und sozialen Beziehungen jenseits der Dichotomie von sozialstaatlichem Sparmodell und idealistisch verklärter Sorgegemeinschaft zu fassen wären (Kapitel 4).

\section{Die ,demenzfreundliche' Gesellschaft: Zum Verhältnis von Sozialstaat und Sorgegemeinschaft}

Die steigende Nachfrage nach medizinischen und pflegerischen Versorgungsleistungen steht seit Jahren im Mittelpunkt eines demografischen Krisendiskurses, der vor den sozialen und ökonomischen Folgen einer globalen alternden Gesellschaft warnt (Statistische Ämter des Bundes und der Länder 2010, Leicht/König 2012). Besonders Demenz wurde hier seit den 1980er Jahren gleichsam zum Inbegriff dieser Krise (Innes 2009, Lane/ McLachlan/Philip 2013). Sie ist zur zentralen Metapher einer ,apokalyptischen Demografie“ (Robertson 1990) geworden, mit der sich die Vulnerabilität des Alter(n)s sowie die damit verbundene prognostizierte Kostenexplosion in 
den Versorgungs- und Pflegeleistungen auf besonders drastische Weise kommunizieren lässt (Wetzstein 2010: 55). Vor diesem Hintergrund forderte das Europäische Parlament 2011 die Mitgliedsstaaten der EU auf, „spezifische nationale Pläne und Strategien für die Alzheimer-Krankheit aufzustellen, um den Folgen von Demenzerkrankungen für die Gesellschaft und das Gesundheitswesen Rechnung zu tragen und Dienstleistungen und Unterstützung für Menschen mit Demenzerkrankungen und ihre Familien bereitzustellen" (zit. nach Kirchen-Peters/Hielscher 2012: 5).

Im Vergleich zu anderen EU-Mitgliedsstaaten setzt die gezielte sozialpolitische Steuerung der Versorgungsstrukturen und Unterstützungsnetzwerke für Menschen mit kognitiven Beeinträchtigungen in Deutschland verhältnismäßig spät ein (Kirchen-Peters/Hielscher 2013: 22). Im Herbst 2012 wird von den Bundesministerien für Gesundheit (BMG) und für Familie, Senioren, Frauen und Jugend (BMFSFJ) die „Allianz für Menschen mit Demenz" gegründet. Die Arbeitsgruppe, die unter Beteiligung verschiedener Organisationen und Verbände einen nationalen Demenzplan entwickeln soll, ist Bestandteil der sogenannten ,Demografiestrategie ${ }^{6}$, mit der die Bundesregierung den demografischen Wandel politisch zu gestalten sucht (Bundesministerium des Inneren o. J.). Die Allianz verfolgt das Ziel, „die öffentlichen Stellen und die Organisationen der Zivilgesellschaft einschließlich der Selbsthilfe zusammen [zu führen], die sich auf Bundes-, Landes- und kommunaler Ebene für Menschen mit Demenz engagieren“; es gehe darum, „die Kräfte aller Verantwortlichen“ zu bündeln (BMFSFJ/BMG 2014: 4). Auffällig ist die zentrale Bedeutung, die zivilgesellschaftlichen Akteur_innen im Rahmen dieses Modells geteilter Verantwortung zukommt: Um Menschen mit kognitiven Veränderungen eine umfassende Teilhabe am gesellschaftlichen Leben zu ermöglichen, sei „Unterstützung durch nachbarschaftliche Hilfe“ gefragt; „neue Formen des Miteinanders“ müssten entwickelt und „bürgerschaftliches/ehrenamtliches Engagement“ weiter ausgebaut werden“ (ebd.: 11). Es ist von „sorgenden Gemeinschaften“ (ebd.) die Rede, die Betroffene und ihre Angehörigen in der Bewältigung der alltäglichen Herausforderungen unterstützen sollen.

Das schillernde Schlagwort der „sorgenden Gemeinschaft“ hat seit einigen Jahren Konjunktur in pflege- und seniorenpolitischen Papieren der Bundesregierung (BMFSFJ 2012: 115, Haubner 2017) und wird „von der Bundeskanzlerin bis zu Stadtplanern“ (Klie 2014: 113) aufgegriffen. Vorläufiger Höhepunkt dieser Entwicklung ist der Siebte Altenbericht, der schwerpunktmäßig die Frage behandelt, wie „Sorge und Mitverantwortung in der Kommune" staatlich unterstützt und die Rahmenbedingungen für den „Aufbau und [die] Sicherung zukunftsfähiger Gemeinschaften“ verbessert werden können (Siebte Altenberichtskommission 2016). Der Sachverständigenrat fordert in dem Bericht eine an den Prinzipien von Subsidiarität und Wohlfahrtspluralismus ausgerichtete „Gestaltung von Pflege- und Sorgeaufgaben" im Sinne des Leitbilds einer Caring Community (ebd.: 194). Zwei Gestaltungsebenen werden hierbei miteinander verknüpft: (1) Verwaltungstechnisch wird die sorgende Gemeinschaft mit der Kommune bzw. dem Stadtbezirk als zuständiger Gebietskörperschaft identifiziert, der daher mehr Gestaltungskompetenzen und finanzielle Mittel zu erteilen seien. Ihr obliege die Bereitstellung des infrastrukturellen Rahmens für (a) die 
Koordination der unterschiedlichen (öffentlichen, privatwirtschaftlichen, zivilgesellschaftlichen) Akteure in der Kommune, die zur Sorge-/Pflegearbeit beitragen und (b) die Mobilisierung und Erleichterung ehrenamtlicher Arbeit. (2) Moralisch und lebensweltlich wird die sorgende Gemeinschaft mit einem solidarischen Zusammenhang von Verwandten, Nachbar_innen und Stadtteilbewohner_innen identifiziert, die auf Basis gegenseitiger Wertschätzung füreinander Verantwortung übernehmen.

\section{Caring Communities - vom Bewegungs- zum Staatskonzept?}

Die Vorstellung von einer sorgenden Gemeinschaft hat eine verzweigte Genealogie mit unterschiedlichen Entstehungskontexten und Bedeutungen (Klie 2014: 113ff.). Der angelsächsische Kommunitarismus, das christliche Gemeindeverständnis und die Debatte über die republikanischeWiederbelebung (Enquete-Kommission 2003) eines von bürgerschaftlichem Engagement getragenen „fürsorgende[n] Gemeinwesen[s]“ (Störmer 2002: 27) können als wichtige Stichwortgeber für ihre Popularisierung betrachtet werden. Seit den 1980er Jahren tauchen kommunitaristische Diskurse verstärkt im Kontext bürgerrechtlicher Bewegungen und der Inklusionsdebatte in der Sozialen Arbeit auf. Konzepte wie ,community care ${ }^{6}$, ,supported living ' und ,enabling environment' gewinnen an Einfluss in der Behindertenarbeit, die sich an Enthospitalisierung, Selbstbestimmung und sozialer Teilhabe von Behinderten orientiert (Priestley 1999, Aselmeier 2007). Im Kontext der humanistisch ausgerichteten Hospiz- und Alzheimerbewegungen nimmt die Idee der sorgenden Gemeinschaften seit den 1990er Jahren in Debatten über ,palliative care ‘ und ,compassionate cities' Gestalt an (Kellehear 2005, Klie 2014). Die Ansätze zielen darauf ab, Altern, Sterben und Tod zu enttabuisieren und der Dominanz des häufig als inhuman charakterisierten professionellen Versorgungsprinzips zu entziehen, das zu unflexibel sei, um adäquat auf individuelle Bedürfnisse einzugehen. Die Unterstützung und Begleitung von Menschen am Lebensende wird stattdessen als humanitäre Aufgabe von „anteilnehmenden Gemeinschaften“ redefiniert (Wegleitner et al. 2015). Die Caring Community unterscheidet sich dabei konzeptuell von einer durch den Sozialversicherungsstaat konstituierten ,nationalen Solidargemeinschaft'. Sie „beginnt vor der Haustür, im Quartier, in der Kommune“ und setzt auf die unmittelbare „Mitverantwortlichkeit“, „Mitschwingungsfähigkeit“ und „Empathiefähigkeit“von Nachbar_innen und Gemeindemitgliedern, die sich füreinander interessieren (Klie 2014: 118). Auch die Diskussion über eine ,demenzfreundliche 'Gesellschaft ist durch solche bürgerrechtlichen und humanistischen Impulse stark geprägt, wie später noch deutlich werden wird. Es gehe um die Entwicklung einer praktisch werdenden und örtlich verankerten Sorgekultur, die das „Recht auf Demenz als Lebensform“ anerkennt, wie es Thomas Klie, Mitglied der Sechsten und Siebten Altenberichtskommission, als einer der prominentesten Protagonist_innen der Caring Community im DAK-Pflegereport 2017 festhält (Pflegereport 2017: XII).

Im Kontext der erwähnten Demografiestrategie der Bundesregierung wird der bürgerrechtliche und humanistische Diskurs über sorgende Gemeinschaften nun seit einigen Jahren verstärkt aufgegriffen. Die gemeinwesenbasierte Unterstützungs- und Fürsorgebereitschaft wird hierbei zum 
Gegenstand einer sozialpolitischen Potenzial- und Nutzenanalyse gemacht (BMFSFJ 2012: 115). Wohlfahrtsstaatstheoretisch betrachtet steht das neue Leitbild der Caring Communities keinesfalls lediglich für den Versuch, öffentliche Infrastrukturen und Dienstleistungen durch bürgerschaftliches Engagement zu ergänzen. Vielmehr deutet die Konjunktur des Konzepts in sozialpolitischen Programmen auf eine Transformation der Beziehung zwischen Sozialstaat, Individuum und Gemeinschaft hin, die sich als Wandel vom „Wohlfahrtstaat zur Wohlfahrtsgesellschaft“ (Rodger 2000) charakterisieren lässt. Dem Sozialstaat kommt in einer solchen dezentralen, unter kommunaler Federführung gestalteten Wohlfahrtsgesellschaft nicht mehr vorrangig die Rolle eines Versorgers, sondern eines Ermöglichers zu (Rose 2000), der die pluralen und informellen Unterstützungsnetzwerke in den Nachbarschaften und Gemeinden monetär und infrastrukturell stabilisiert (vgl. Siebte Altenberichtskommission 2016).

Die Einführung und der Ausbau der Pflegeversicherung seit 1995 bringen diesen Wandel paradigmatisch zum Ausdruck. Zwar handelt es sich hierbei um eine zentralisierte Transferleistung, doch wird nun erstmals die Förderung und Gestaltung - und nicht bloß stillschweigende Voraussetzung - der informellen Unterstützungs- und Fürsorgebeziehungen in Familie und sozialer Umgebung in ein Sozialversicherungsmodell integriert (Brömme 1999: 3). Denn ,Cash-for-care'-Programme wie das Pflegegeld oder die Verhinderungspflege sind explizit nicht als bedarfsdeckende Grundversorgung konzipiert, sondern als monetäre Transferleistungen, die die Pflegebereitschaft von Angehörigen und Nachbar_innen unterstützen sollen (Gerlinger/Röber 2012).[3]

Die hohe Resonanz, die der Ruf nach einer „Sorgekultur“ und einem „Aufbruch“ in die „sorgende Gesellschaft“ (Klie 2014) in der staatlichen Pflege- und Seniorenpolitik erfährt, lässt sich erst mit Blick auf die wohlfahrtsstaatlichen und polit-ökonomischen Rahmenbedingungen der gesellschaftlichen Organisation der Altensorge erklären, auf die im Folgenden näher eingegangen werden soll.

\section{Gemeinsam aus der Altenpflegekrise? Wohlfahrtsstaatliche und polit-ökonomische Rahmenbedingungen ,sorgender Gemeinschaften ${ }^{6}$}

Traditionell insbesondere weiblichen Familienangehörigen zugewiesen, war die Altenpflege als Arbeitsbereich schon immer überwiegend informell organisiert (Theobald 2008: 258, Knijn/Kremer 1997). Dies gilt auch für die Pflege von Menschen mit Demenz, die zu zwei Dritteln in der häuslichen Umgebung durch mehrheitlich weibliche Verwandte geleistet wird (BMFSFJ 2002: 201). Dieses Modell gerät jedoch zunehmend in Bedrängnis (Lutz 2012); Schätzungen gehen von einem Rückgang des informellen familiären Pflegevolumens um 30 bis 40 Prozent bis 2050 aus (Maier 2008). Neben demografischen Entwicklungen sind hierfür maßgeblich die Transformation der Familien- und Geschlechterverhältnisse sowie ein Wandel der Produktions- und Akkumulationsverhältnisse verantwortlich. Die weitflächige Umstellung vom Ein-Ernährer-zum Adult-worker-Modell, das (im Einklang mit Forderungen der liberalen Frauenbewegung) eine verstärkte 
Integration von Frauen in den Erwerbsarbeitsmarkt vorsieht, hat den Familienhaushalt als informelle „Hauptpflegestelle der Nation“ (Siebte Altenberichtskommission 2016: 195) ebenso schrumpfen lassen wie gestiegene Mobilitätsanforderungen und die Pluralisierung von Lebensformen. Beispiele für Letztere sind der Anstieg von Einpersonenhaushalten sowie ein wachsender räumlicher Abstand zwischen den Familiengenerationen (ebd.: 49).

Die Umdeutung und Umgestaltung der zivilen Bürgergesellschaft in eine Care-Gemeinschaft wird von kritischen Sozialwissenschaftler_innen vor diesem Hintergrund als Strategie des aktivierenden Sozialstaates gefasst, der den Rückgang familiärer Care-Ressourcen durch den Rückgriff auf andere Formen unbezahlter Care-Arbeit zu kompensieren sucht (van Dyk 2016; 2017, Haubner 2017, Graefe 2010). Um die Systematik und Funktion der vermehrten Indienstnahme ehrenamtlicher Tätigkeiten in den Kommunen und Nachbarschaften zu unterstreichen, sprechen Silke van Dyk (2017) und Tine Haubner (2017: 450ff.) gar von der Entstehung einer neuen polit-ökonomischen Formation, die sie „Community-Kapitalismus“ (van Dyk 2017: 1; Haubner 2017: 450ff.) nennen. In Zeiten einer Erschöpfung der Familie als Versorgungssystem avanciere die solidarische Gemeinschaft zum neuen „Lebensexelier“ des Kapitalismus (van Dyk 2017: 3).[4] Die Indienstnahme der Care-Gemeinschaft verstärke hierbei die Deregulierung und Deprofessionalisierung des ohnehin abgewerteten Bereichs der Pflege- und Care-Arbeit (Haubner 2017: 20).

Die Transformation von Wohlfahrtsstaatlichkeit im Community-Kapitalismus stelle zudem einen Angriff auf erkämpfte soziale Rechte wie das Recht auf ausreichende soziale Absicherung dar (van Dyk 2017; 2016). Dies geschehe insbesondere mittels einer moralisierenden Individualisierung: Die Solidarität der Mitglieder einer Gesellschaft werde im Rahmen der Gemeinschaftssemantik nicht mehr sozialstaatlich vermittelt und abgesichert - durch einen ,anonymen Ausgleichsmechanismus“, der „an die Stelle klassischer gemeinschaftsbasierter Solidaritätsformen“ trete -, sondern an die Existenz konkreter und personalisierter Beziehungen zwischen Individuen gekoppelt (van Dyk 2017: 7, vgl. auch Rose 2000: 77). Das ,Versicherungsprinzip' des Sozialstaats sei daher - bei aller notwendigen Kritik an seinen ungleichheitsstiftenden Implikationen - gegen eine Reziprozität verlangende Moral des Gemeinsinns in Schutz zu nehmen. Denn es handele sich um „eine wesentliche, wenn auch nie umfassend realisierte Errungenschaft der Moderne, dass ein Mensch nicht gemocht oder als besonders (klug, interessant, einzigartig) anerkannt werden muss, um ein Recht auf (soziale, politische, bürgerliche) Rechte zu haben“ (van Dyk 2016: 251). Allerdings wäre hier relativierend hinzuzufügen, dass sämtliche, auch stärker institutionalisierte, Care-Beziehungen durch einen Care-Parochialismus geprägt sind, der Care-Nehmende bevorteilt, denen sich Carearbeiter_innen näher und verbundener fühlen (Tronto 2010: 161). Der Parochialismus-Einwand muss daher noch in keine prinzipielle Absage an vergemeinschaftete Sorge-Arrangements münden, sondern kann ebenso als allgemeine Aufforderung zu einer demokratischen und sozial gerechten Ausgestaltung von Caring Communities verstanden werden.

Die skizzierten Kritikperspektiven warnen dennoch zu Recht vor einem idealisierenden, romantisierenden Blick auf die Idee und Praxis sorgender 
Gemeinschaften. Sie zeigen die Widersprüchlichkeit kollektiven Sorgens in Zeiten seiner sozialstaatlichen Instrumentalisierung und Inwertsetzung auf und bilden damit ein wichtiges Korrektiv zu den Altenberichten und Demenzplänen der Bundesregierung sowie den Papieren von Alzheimergesellschaften, Pflegestützpunkten und bürgerschaftlichen Initiativen, in denen die sorgende Zivilgesellschaft häufig ,in weich gezeichnetem Licht als Quelle von ,Würde‘, ,Wertschätzung' und ,neuer Alterskultur““ (Graefe 2010: 255) erscheint. Allerdings lässt sich die Widersprüchlichkeit der Idee und Praxis sorgender Gemeinschaften umgekehrt auch nicht in einer ausbeutungsund sozialstaatszentrierten Perspektive auflösen. Gemeinschaftliche Sorgearrangements würden damit auf Aspekte ihrer staatlichen Kooptierung und ökonomischen Vernutzung reduziert. Der Blick auf darüber hinausweisende emanzipatorische Potenziale einer kollektiven Organisation von Sorgearbeit, wiesie in bürgerrechtlichen Forderungen nach einem Weniger an,Versorgung und einem Mehr an sozialer Inklusion aufscheinen, wäre so verstellt. So erschöpft sich eine bedarfsgerechte Gestaltung von Sorgeverhältnissen nicht im Volumen staatlicher Transferleistungen und professioneller Pflegedienstleistungen, sondern umfasst zugleich lebensweltliche Formen der physischen, emotionalen und psychischen Bedürfnisbefriedigung. Wenn apersonale gesellschaftliche Solidaritäten gegenüber gemeinschaftsförmigen personalisierten Solidaritäten favorisiert werden, stellt sich die Frage, wie Bedürfnisse nach fürsorglichen Beziehungen befriedigt werden sollen, ohne den wohlfahrtsstaatlichen Status quo zu reproduzieren, der diese Aufgabe an private Unterstützungssysteme (Familie, Liebesbeziehungen, Freundschaften) delegiert.

Durch eine Erweiterung des wohlfahrtsstaatstheoretischen und polit-ökonomischen Analyserahmens um feministische und disability-theoretische Perspektiven auf Citizenship, Care und Demenz soll die Untersuchungsperspektive daher im Folgenden für emanzipatorische und transformative Potenziale gemeinschaftlicher Sorgearrangements analytisch geöffnet werden. Als empirisches Fallbeispiel dient mir hierfür das bürgerrechtliche Konzept der ,demenzfreundlichen Kommune‘.

\section{Ein ,neues soziales Miteinander'? - Emanzipatorische und transformative Potenziale der ,demenzfreundlichen Kommune ${ }^{6}$}

Bereits Jahre bevor die Bundesregierung die Verbesserung der Lebens- und Versorgungssituation von Menschen mit Demenzdiagnose zum expliziten (Teil-)Ziel ihrer sozialpolitischen Gestaltung sorgender Gemeinschaften erklärte (s. Kapitel 1), reiften Ideen und erste Handlungsansätze zur Umsetzung von ,demenzfreundlichen Kommunen ' im Umkreis professioneller Akteur_innen und zivilgesellschaftlicher Initiativen wie AlzheimerGesellschaften heran (Rothe/Kreutzner/Gronemeyer 2015). 2006 gründet sich der gemeinnützige Verein „Aktion Demenz. Gemeinsam für ein besseres Leben mit Demenz“, dessen Aufgabe darin besteht, „demenzfreundliche Kommunen" als noch zu verwirklichendes Zukunftsprojekt anzustoßen, mit dem „Demenz gleichsam aus dem abgeschotteten Dasein einer PariaThematik in die Mitte der Gesellschaft und mitten in das Gemeinwesen“ 
hineingetragen werden solle (Kreutzner 2015: 253). Der zugrunde gelegte Leitsatz lautet, dass „Menschen mit Demenz nicht nur pflege- und hilfebedürftige Kranke, sondern wie wir Bürgerinnen und Bürger dieses Staates und unserer Kommunen sind“ (Rothe 2015: 47). Hieraus leiten sich verschiedene Fragen ab, die sich jedes Gemeinwesen zu stellen habe:

„Was muss in einem Dorf, einer Stadt und/oder einer Region geschehen, damit sich Menschen mit Demenz möglichst wohlfühlen können? Was kann getan werden, damit Räume und Möglichkeiten des Kontakts und der Begegnung zwischen Menschen mit Demenz und anderen Mitgliedern in einem Gemeinwesen entstehen können? Damit von einer Demenz Betroffene und ihre Angehörigen nicht alleine gelassen oder gar isoliert werden? Damit Menschen mit einer Demenz so lange wie möglich in dem ihnen vertrauten Umfeld leben können?" (Aktion Demenz e.V. 2009: 4)

Im Jahr 2008 begann Aktion Demenz e.V. im Rahmen des von der Robert Bosch Stiftung finanzierten Förderprogramms „Menschen mit Demenz in der Kommune“ lokale Projekte und Initiativen anzustoßen, die die Lebensqualität von Menschen mit kognitiven Beeinträchtigungen vor Ort zu verbessern suchen.[5] Insgesamt 78 Projekte werden in diesem Rahmen bundesweit gefördert. Sie weisen hinsichtlich der Trägerschaft (u. a. Stadtverwaltungen, Wohlfahrtsverbände, Kirchengemeinden, Vereine, ehrenamtliche Nachbarschaftsinitiativen, Pflegeeinrichtungen) und Ausrichtung (z. B. kulturelle, sportliche und künstlerische Angebote, Begegnungsstätten, Vernetzungs-, Aufklärungs- und Sensibilisierungsarbeit) eine hohe Heterogenität auf; auch die Anteile von Professionellen und Ehrenamtlichen an der Projektarbeit variieren erheblich. Gemein ist den meisten Projekten ein Fokus auf soziale Inklusion und Teilhabe, auf Mitbestimmung, Gemeinsinn und Solidarität. Sie beteiligen sich damit an dem von „Aktion Demenz“verfolgten Ziel, das Thema der Demenz aus dem engen Rahmen der Versorgungssemantik herauszulösen und als eine gesamtgesellschaftliche bürgerrechtliche Problematik zu adressieren.

\section{Das Citizenship-Demenzmodell: Jenseits des Patient_innenstatus}

Menschen mit Demenzdiagnoseals Bürger_innen zu betrachten, diepolitische, zivile und soziale Rechte haben, richtet sich gegen den Reduktionismus klinisch-medizinischer und versorgungslogischer Demenzmodelle, die Personen mit kognitiven Veränderungen mit dem passiven Status ,Patient_in“ und ,Leistungsempfänger_in“ identifizieren (Kreutzner 2015: 241, Wißmann 2017a: 24f.). Wenn die Initiator_innen des Programms der „Demenzfreundlichen Kommunen“ eine „neue Kultur des Umgangs mit Demenz“ (Kreutzner 2015: 238) fordern, geht es ihnen insbesondere auch um das Verlernen eines kulturell sedimentierten Demenzbildes, das Betroffene darüber definiert, was sie verloren haben und nicht (mehr) fähig sind zu tun (Bartlett/ O'Connor 2010: 18). Das dominante Demenzbild interpretiert die Demenz als kontinuierlichen Zerfallsprozess, als Verlust von Identität und Selbst, der sinnvolle und reziproke Beziehungen verunmöglicht. Demenz gilt dann als Hinderungsgrund für soziales Engagement, kulturelle Aktivität und politische 
Teilhabe. In Bezug auf Pflege- und Unterstützungsarrangements begünstigt das defizitäre Demenzmodell eine Haltung, die mit Viktimisierung, Bevormundung und Kontrolle einhergeht (Jones/Higgs 2010, Katz/Westphal 2004, Rohra 2012). Dies, so das Argument einer citizenship-orientierten Position, trage zur gesellschaftlichen Marginalisierung, Ausgrenzung und Passivierung von Menschen mit kognitiver Beeinträchtigung bei.

Die Citizenship-Debatte wirft hier die Frage nach den Machtverhältnissen auf, die das Leben und Erleben von Menschen mit Demenzdiagnose durchziehen (Bartlett/O'Connor 2010: 38). Durch die Stärkung der politischen und sozialen Rechte von Betroffenen soll erreicht werden, dass diese innerhalb der konkreten Care-Beziehungen in ihrem Alltag - mit Ärzt_innen, Pfleger_innen, aber auch Angehörigen - weniger Ohnmacht erfahren (vgl. Proctor 2001, Bartlett/O'Connor 2010: 111). Formen der Entmündigung und Passivierung von Menschen mit kognitiven Veränderungen werden aber nicht lediglich innerhalb der konkreten Beziehung zur unterstützenden/pflegenden Person, sondern auch mit Blick auf die organisationsbezogenen, institutionellen und wohlfahrtsstaatlichen Rahmenbedingungen thematisierbar, die das Leben mit Demenz strukturieren. So lässt sich beispielsweise fragen, welche institutionalisierten Normen und Versorgungsstrukturen mit dafür verantwortlich sind, dass Menschen mit dementiellen Veränderungen Grundrechte, wie das Recht auf gesellschaftliche und politische Partizipation, nicht oder nur gegen gravierende Widerstände wahrnehmen können.[6]

Anders als das klinischmedizinische und versorgungslogische Demenzmodell schreibt die Citizenship-Perspektive Personen mit kognitiven Veränderungen ein aktives Gestaltungspotenzial hinsichtlich der sozialen Beziehungen und Räumezu, an denen siepartizipieren. Wieweit sich dieses Potenzial entfalten lässt, etwa hinsichtlich der Wohnortwahl, der Bewegungsfreiheit oder der Wahl der Pflegepersonen, hänge weniger von den individuellen kognitiven Fähigkeiten einer Person ab, sondern davon, ob und wie weitgehend rechtliche, politische und lebensweltliche Rahmenbedingungen geschaffen werden, in denen Menschen mit Demenzdiagnose ihre Rechte als Bürger_innen wahrnehmen können (Bartlett/O’Connor 2010, Kontos et al. 2017). Die ,demenzfreundliche Kommune“ will diese Bedingungen schaffen, indem sie Kooperation und Relationalität zur sozialen Voraussetzung und Ermöglichung eines doing citizenship als interdependeter Praxis erklärt. Die Teilhabe und Inklusion von Menschen mit kognitiven Veränderungen an der Stadtgesellschaft lasse sich hierbei nicht schlicht über soziale Transfer- und Dienstleistungen bewerkstelligen, sondern könne nur durch eine systematische Kooperation der zivilen Kräfte mit den Betroffenen in den Kommunen gelingen, wie nachfolgend erläutert werden soll.

\section{Caring for Citizenship: Kollektives Sorgen als politische Wiederbelebung der Zivilgesellschaft}

Die ,demenzfreundliche Kommune ' wird als sozialer Nahraum gefasst, in dem Teilhabe und Wohlbefinden von Menschen mit demenziellen Veränderungen durch eine Transformation der sozialen Beziehungen zu den anderen Stadtbewohner_innen ermöglicht werden soll. Es sollen Schnittstellen im urbanen Raum entstehen, an denen sich Menschen mit und ohne kognitive 
Beeinträchtigungen jenseits professioneller und verwandtschaftlicher CareBeziehungen begegnen. Menschen mit Demenzdiagnose sollen hierbei als aktive Partner_innen der gemeinsamen Beziehungsgestaltung - und als Expert_innen in eigener Sache erlebt werden (vgl. Rothe 2015, Demenz Support Stuttgart 2013; 2017, Rohra 2012). Sie seien darüber hinaus aktiv an der kommunalen Stadtplanung zu beteiligen. Dies betrifft Aspekte, die direkt mit der Beeinträchtigung verknüpft sind: vom Schaffen ausreichender Begegnungs- und Unterstützungsmöglichkeiten über die Planung von Orientierungssymbolen und sicheren Straßenübergängen bis zur verbesserten „Versteh- und Bedienbarkeit technischer Apparaturen, wie beispielsweise Fahrkartenautomaten“ (Wißmann 2017b: 122). Aber auch die Beteiligung an Entscheidungsprozessen, die mit ihrer kognitiven Beeinträchtigung in keinem Zusammenhang stehen, sondern sie als Stadtteilbewohner_innen betreffen, müsse gewährleistet werden, vom Bau eines Einkaufszentrums bis zur Gestaltung von Park- und Grünflächen.

Damit eine solche Partizipation möglich werde, müssten Menschen ohne Beeinträchtigungen ihre Kommunikations- und Interaktionsgewohnheiten verändern. Es bedürfe geeigneter Methoden und Praktiken, die dieveränderten kognitiven Voraussetzungen der Betroffenen berücksichtigen. Anstelle von Bürger_innenversammlungen werden so zum Beispiel direkte Befragungen im Kontext gemeinsamer Stadtspaziergänge erprobt. Auch mit veränderten Workshopformaten, in denen weniger argumentative als sinnlich-körperlichexpressive Ausdrucksweisen im Mittelpunkt stehen, wurde im Rahmen partizipativer Community-Praxis bereits gearbeitet (Demenz Support Stuttgart 2017). Der relationale Begriff von Citizenship (Brannelly 2016, Kontos/Miller/Kontos 2017), der die Idee von,demenzfreundlichen Kommunen' anleitet, indem Interdependenz und das alltägliche Aufeinanderverwiesensein an die Stelle des Autonomismus traditioneller CitizenshipKonzepte rückt, ist demnach zugleich ein verkörperter (Kontos 2012). Er geht auf Abstand zum Kognitivismus vorherrschender Demenzmodelle und fokussiert „die Wiederaneignung und Eröffnung leiblicher und sinnesorientierter Erfahrungs- und Interaktionsräume“, die „neue Möglichkeiten der Welterfahrung, der Kommunikation und der gesellschaftlichen Teilhabe“ (Wißmann/Gronemeyer 2008: 74f.) erschlössen.

Ein relationaler und verkörperter Begriff von Citizenship ist unmittelbar anschlussfähig an feministische Care-Ethiken, die Interdependenz, Fürsorglichkeit und eine Sorge umeinander als konstitutiv für politisch-demokratische Systeme sowie eine an sozialer Gerechtigkeit und Gleichheit orientierte Gesellschaft begreifen (Tronto 2013, Sevenhuijsen 1998). Die feministische Intervention besteht darin, Care und Citizenship integral ineinander zu verschränken: Ob Bürger_innen in die Lage versetzt werden, sozial und politisch zu partizipieren und ob sie ihre Rechte und wechselseitige Verantwortung füreinander innerhalb einer politischen Gemeinschaft wahrzunehmen vermögen, hänge von der Existenz und dem Erhalt situierter, fürsorglicher sozialer Beziehungen ab, auf die jedes Leben angewiesen sei (Kontos/Miller/Kontos 2017: 183).

Ein Gemeinwesen, das solche Beziehungen ausreichend bereitstellt, existiere aber nach Dafürhalten der Protagonist_innen der ,demenzfreundlichen Kommunen“ in der Gegenwart nicht. Die Demenz gebe daher 
auch Gelegenheit für die politische und soziale Wiederbelebung eines solidarischen, demokratischen und sorgsamen Gemeinwesens, „in der die Hilfe, die Menschen brauchen, nicht ausschließlich von bezahlten Dienstleistern erwartet wird. So verstanden, geht es bei ,demenzfreundlichen Kommunen' um das Erfinden eines neuen sozialen Miteinanders" (Rothe/ Kreutzner/Gronemeyer 2015: 14). Es solle ein gemeinsames, kommunales ,Wir ' geschaffen werden, das sich städtischen Raum aneignet beziehungsweise dort wiederherstellt, wo das soziale Leben durch eine „konsumistische Monokultur" überdeterminiert und „kommunikativ entleert“ wurde (Rothe/ Kreutzner/Gronemeyer 2015: 33). Bürgerschaftliches Engagement sei daher auch nicht „Notlösung“ (Wißmann/Gronemeyer 2008: 86), sondern Motor eines sozialen Wandels hin zu einer Gesellschaft, die die Notwendigkeit hervorhebt, „für andere da zu sein“ (Klie 2014: 130) und „auf andere zu zählen“ (Precarias a la deriva 2011: 112).

\section{4. ,Demenzfreundliche Kommunen' - sozialstaatliche Sparressource oder Modell für eine emanzipatorische Sozialpolitik von unten?}

In der bürgerrechtlichen Diskussion über ,demenzfreundliche Kommunen wird immer wieder hervorgehoben, dass es den Protagonist_innen nicht darum gehe, „Politik aus ihrer [...] Verantwortung zu entlassen“, ausreichend soziale Dienstleistungen und Infrastrukturen zur Verfügung zu stellen (Rothe 2015: 43).

„Es handelt sich nach unserem Verständnis nicht um einen weiteren Baustein im Ausbau einer Versorgungslandschaft, bei der lediglich die kommunale Struktur als neue Dienstleistungsebene zwischen Pflegeheim und Familie eingezogen wird. Es handelt sich auch nicht um eine mehr oder weniger trickreiche Strategie, den Sozialstaat zu entlasten, in dem man nicht mehr bezahlbare Versorgungsleistungen an ehrenamtlich und damit kostengünstig arbeitende Substrukturen abgibt“ (Gronemeyer 2011: 6).

Dass Idee und Praxis einer von bürgerschaftlichem Engagement getragenen ,demenzfreundlichen Kommune unter den gegebenen Rahmenbedingungen (Kapitel 2) mit dem weiteren Rückbau der öffentlichen Daseinsvorsorge sowie einer ,von oben' gesteuerten Responsibilisierung zivilgesellschaftlicher Care-Ressourcen einhergehen könnten, ist den Akteur_innen hierbei durchaus bewusst: Was als „bürgerschaftlicher Aufbruch“ von unten, als „Graswurzelbewegung“ für „eine neue Konvivialität“ und „eine Revolution des Zusammenlebens" (Rothe/Kreutzner/Gronemeyer 2015: 30) in den Gemeinden gedacht war, sei der Gefahr ausgesetzt, von der „Gesundheitsadministration“ (ebd.) des Bundes instrumentalisiert und angeeignet zu werden.

Dieser Prozess habe mit dem aus öffentlichen Mitteln finanzierten und ministerial gesteuerten Vorzeigeprojekt der Bundesregierung, der „Lokalen Allianz für Demenz“, bereits konkret Form angenommen. Mit dem Programm, das nach eigenen Angaben die „Potenziale von Bürgerinnen und Bürgern, Vereinen, Gewerbe, Bildungseinrichtungen, Politik und Kommunalverwaltung“ bündeln und vernetzen will (Lokale Allianz für 
Demenz o. J.: o. S.), wurden von 2012 bis 2017 insgesamt 500 lokale Projekte über zwei Jahre mit jeweils 10.00o Euro gefördert, darunter Diakonische Werke, Hospitzdienste, Stadtteilzentren, Mehrgenerationenhäuser und (häufig zur Tagespflege genutzte) gerontopsychiatrische Kliniken. In diesem Rahmen sind in vielen Städten Trommel- und Kochgruppen oder Tanzcafés entstanden, Demenzlotsen-Projekte wurden ausgebaut und transkulturelle Initiativen für Migrant_innen wurden angestoßen.

Die Akteur_innen der ,demenzfreundlichen Kommunen' betrachten solche und andere Initiativen, von oben' mit Skepsis. Ihre Kritik an einer Institutionalisierung und Regulierung gemeinschaftlicher Sorge- und Unterstützungspraktiken fußt - neben der Instrumentalisierungsproblematik - auf zwei Argumenten: Erstens würde eine Regulierung sorgender Gemeinschaften auf Landes- und Bundesebene den radikaldemokratischen Kern ,demenzfreundlicher Kommunen' aufweichen, sofern Bürger_innen mit und ohne Beeinträchtigungen nicht mehr unmittelbar Einfluss auf Form und Inhalt der verfügbaren Angebote und Leistungen hätten. Zweitens sei der citizenship-basierte Leitgedanke von Partizipation und Inklusion in den staatlich-administrativ verantworteten Programmen nicht oder kaum noch erkennbar. Vielmehr würde eine „Demenzparallelwelt mit ihren Alzheimertanzcafés, Demenzgottesdiensten und anderen Spezialangeboten“ (Wißmann 2017a: 25) errichtet, die dem klassischen Versorgungsmodell verhaftet bliebe. Diese „Separierung in speziellen Angebots- und Institutionsformen“ (Wißmann 2016: 29) finde ihre Zuspitzung im Konzept der ,Demenzdörfer‘, wo „Demenzbetroffene in großer Zahl am Rande der Städte in speziellen geschlossenen Arealen untergebracht und betreut werden“ (ebd. 31). Die ,demenzfreundliche Kommune ist aus Sicht ihrer Befürworter_ innen gerade das Gegenmodell zur Schaffung solcher exklusiven Räume. Es gehe gerade darum, die Demenz in die Gesellschaft herein zu holen und als „eine mögliche Seinsform im Alter“ (Wißmann/Gronemeyer 2008) anzunehmen, mit der die Gemeinschaft und jede_r Einzelne lernen müsse, umzugehen (Rothe 2015: 99).

Auffällig an der Debatte zur ,demenzfreundlichen Kommune‘ ist, dass die Gefahr der sozialstaatlichen Instrumentalisierung zivilgesellschaftlicher Unterstützungs- und Sorgestrukturen einerseits erkannt wird, andererseits aber Ratlosigkeit vorzuherrschen scheint, was dem entgegenzusetzen wäre. „Ob der Rückzug des Sozialstaates ökonomisch notwendig ist oder nicht“, heißt es etwa bei Gronemeyer und Wißmann, „er findet nun einmal statt. Und die Bürgergesellschaft muss darauf reagieren, so sie Menschen mit Demenz [...] nicht einfach der Vernachlässigung preisgeben will“ (Wißmann/ Gronemeyer 2008: 104). Die politische oder zumindest rhetorische Strategie von Protagonist_innen der,demenzfreundlichen Kommune' besteht wie erläutert darin, den „kränkelnde[n] Sozialstaat“ (ebd.) als Chance für eine Repolitisierung und Resozialisierung der Bürger_innengesellschaft zu betrachten. Dies erweist sich aus gesellschaftskritischer Perspektive jedoch als ebenso unbefriedigend wie im Hinblick auf eine emanzipatorische Sozialund Care-Politik.

So geht auch der bürgerrechtliche Ansatz der, demenzfreundlichen Kommunen“ von einer dualistischen Gegenüberstellung von Mikround Makroebene, von sorgender Gemeinschaft und staatlich regulierter 
Daseinsvorsorge aus - allerdings mit umgekehrten Vorzeichen als die in Kapitel 2 skizzierten Kritikperspektiven. Besonders deutlich wird dies dort, wo die Protagonist_innen der,demenzfreundlichen Kommune‘ der nichtkäuflichen, über Solidarität und Gemeinschaftssinn vermittelten, ehrenamtlichen Tätigkeit eine höhere Qualität als der sozialen Dienstleistung beimessen: „Niemals wird die perfekte Dienstleistung das bringen können, was die Menschen mit Demenz am meisten brauchen: Dass einer für sie da ist. Als Du. Als Mensch. Als Gegenüber.“(Gronemeyer 2015: 33)

Warum eine professionelle Pflegekraft, ein ausgebildeter Sozialarbeiter oder eine bei einem gemeinnützigen oder privaten Träger angestellte ,Persönliche Assistenz' nicht in der Lage sein sollte, der Person mit Demenzdiagnose auf Augenhöhe und ,als Mensch' gegenüberzutreten, wird nicht begründet. Ebenso wenig wird nachvollziehbar gemacht, warum nicht auch die öffentliche Daseinsvorsorge prinzipiell demokratisierbar sein und Infrastrukturen sowie Dienstleistungen gewährleisten könnte, die Partizipation, Mitbestimmung und soziale Inklusion ermöglichen.

Jenseits des Dualismus von Gemeinschaft vs. Staat: Zum Emanzipationsund Transformationspotenzial ,demenzfreundlicher Kommunen

Sowohl die in Kapitel 2 skizzierten polit-ökonomischen und wohlfahrtsstaatstheoretischen Kritikperspektiven als auch der bürgerrechtliche und gemeinschaftsaffirmierende Ansatz der ,demenzfreundlichen Kommunen“ bleiben mit ihrer Argumentation einer Logik des Nullsummenspiels verhaftet: Für erstere bedeutet ein Mehr an zivilgesellschaftlicher Verantwortung ein Weniger an sozialstaatlich hergestellter Sicherheit. Für die anderen führt eine Zunahme staatlich gesteuerter Daseinsvorsorge zu einer Abnahme an Teilhabe, Selbstbestimmung und bürgerschaftlicher Solidarität im Alltag.[7] Die Frage lautet, wie diese Nullsummenlogik durch einen theoretischen Rahmen überwunden werden kann, der Verbindungs- statt Trennlinien zwischen den verschiedenen Ebenen sozialer Organisation (Mikroebene, institutionelle Ebene, Makroebene) herstellt. Ein solcher Rahmen würde versuchen, die Stärken beider Perspektiven aufzunehmen, um ihre jeweiligen Verengungen zu korrigieren. Hinweise auf mögliche Anhaltspunkte für ein solches Unternehmen lassen sich, wie abschließend argumentiert werden soll, im Diskurs und Politikfeld der ,demenzfreundlichen Kommunen“ durchaus bereits finden. Im Kontext der politökonomischen Formation des ,Community-Kapitalismus' wären diese Aspekte darauf hin zu betrachten, ob sie auf eine Weise verallgemeinerbar und ausdehnbar sind, dass sie sich einer passivierenden Vereinnahmung und Instrumentalisierung durch den aktivierenden Sozialstaat zu versperren vermögen.

Die Stärken des gemeinschaftsaffirmierenden Ansatzes liegen meines Erachtens in der Politisierung der alltäglichen Sorgepraktiken und -beziehungen, deren Relevanz für gesellschaftliche Transformationsprozesse analytisch aufgewertet wird. Der Demenzaktivismus demonstriert, so wie andere emanzipatorische und bürgerrechtlich orientierte,Gesundheitsbewegungen vor ihm (z. B. die linke Behindertenbewegung und der AIDS-Aktivismus), dass lokale community-basierte Initiativen angesichts eines defizitären öffentlichen und sozialen Unterstützungssystems dazu fähig sind, kollektive 
Strategien der Ent-Prekarisierung zu entwickeln. Indem sie auf Abstand zu den häufig als normierend, diskriminierend und entmündigend empfundenen Formen institutionalisierter wie familiärer Versorgungsstrukturen treten, eröffnen sie einen intermediären Raum für die Entstehung von Neuem: neue Formen des Aushandelns und der Enthierarchisierung von Sozial- und CareBeziehungen; neue Verständnisse von Pflege, Unterstützung und Assistenz, die in dem Moment entstehen, wo herkömmliche Medikalisierungs- und Pathologisierungsformen und die auf ihnen basierenden Pflegekonzepte und Fürsorgepraktiken hinterfragt werden. Soziale Bewegungen haben es auf diese Weise vermocht, sich Epidemien, ,Krankheiten“ oder ,Beeinträchtigungen“ regelrecht anzueignen. Quellen der Isolation, Stigmatisierung und Ausgrenzung wurden in eine Ressource für soziale Bindungen, Empowerment und kollektive Handlungsmacht verwandelt (für den AIDS-Aktivismus vgl. Laufenberg 2014: 325-335).

Auch die ,demenzfreundlichen Kommunen' haben in dieser Hinsicht viel zu bieten: Sie vertreten die Vision, einer anderen Art des Sorgens' auf Augenhöhe und brechen auf diese Weise mit Formen des passivierenden und entmündigenden Versorgungsdenkens. Die ,demenzfreundliche Kommune، weist auf der mikro-sozialen Ebene viele Ansatzpunkte und Schnittstellen für eine gesellschaftliche Transformationsperspektive auf, in der menschliche Bedürfnisse sowie ein Recht auf Teilhabe und Care ins Zentrum einer partizipativen demokratischen Stadtgesellschaft rücken. Die historischen (und immer noch aktuellen) Beispiele des AIDS-Aktivismus und der linken Behindertenbewegung (wie die sogenannte ,Krüppelbewegung') haben deutlich gemacht, dass die gemeinschaftlich-solidarische Einübung anderer Sorgebeziehungen und -praktiken durchaus auf höhere Ebenen sozialer Institutionalisierung übertragbar sind. Sie mündeten hier beispielsweise in die Forderung und partielle Durchsetzung öffentlich finanzierter Dienst- und Transferleistungen, die den spezifischen Lebensumständen und Bedürfnissen der jeweiligen Gruppen besser Rechnung tragen (z. B. Aufnahme der Forderung nach Selbstbestimmung in das SGB IX; Anerkennung der ,Persönlichen Assistenz a als Alternative zur karitativen Hilfe und Pflege; (partielle) Kostenübernahme bei Medikamenten zur Prävention von sexuell übertragbaren Krankheiten, wie aktuell im Falle der HIV-Präexpositionsprophylaxe).

Daher bleibt die ,demenzfreundliche Kommune، hinter ihrem eigenen Anspruch und hinter ihren Möglichkeiten, das soziale Zusammenleben zu „revolutionieren“ (Rothe/Kreutzner/Gronemeyer 2015: 30), zurück, wenn sie das transformatorische Potenzial einer Kommunalisierung des Sorgens lediglich auf einer mikrosozialen und -moralischen Ebene verortet. Denn der an Interdependenz und Angewiesensein orientierte gesellschaftliche Umbau, der die Bedürfnisse und den Bedarf der Menschen nach Unterstützung, Teilhabe und Care ins Zentrum stellt, wird angesichts der begrenzten kommunalen Ressourcen und gemeinschaftlichen Güter ohne eine Ausweitung und Reorganisation der sozialen Daseinsvorsorge nicht möglich sein. Dies hält auch die Siebte Altenberichtskommission (2016) fest, die Bund und Länder auffordert, die „rechtlichen, finanziellen und institutionellen Rahmenbedingungen“ (ebd.: 285) zu schaffen, damit lokale soziale Care-Netzwerke im Alltag nicht nur rhetorisch beschworen, sondern 
von leistungsfähigen und gesetzlich abgesicherten „Altenhilfestrukturen“ (ebd.: 294) getragen werden. Dies beinhalte ein demokratisches Mitspracheund Gestaltungsrecht aller Bürger_innen (ebd.), den Ausgleich sozialer Ungleichheiten - auf Haushalts- wie auf kommunaler und regionaler Ebene (ebd.: 286ff.) - sowie die Ausstattung mit einem „Pflegebudget“, das die individuelle „Wahrnehmung eigener Bedürfnisse und Bedarfe“erlaubt (ebd.: 181).

Die Frage bleibt, wie sich solche und andere durchaus progressive Forderungen, die in aktuellen pflege- und sozialpolitischen Debatten vorgebracht werden, politisch durchsetzen lassen. Im Rahmen einer emanzipatorischen sozialen Infrastruktur- und Care-Politik, die Akteur_innen verschiedener Ebenen zusammenführt (zivilgesellschaftliche, staatliche, gemeinnützige, Expert_innen in eigener Sache etc.), könnten ,demenzfreundliche Kommunen' hier in verschiedener Hinsicht eine bedeutende Rolle spielen. Sie könnten als Stichwortgeberinnen für die Qualitätsverbesserung sowie partizipative und inklusive Ausgestaltung sozialer Dienstleistungen und Infrastrukturen (Aust et al. 2017: 6) fungieren, sofern es nicht nur um den Ausbau, sondern zugleich um einen demokratischen Umbau der öffentlichen Daseinsvorsorge geht. Sie könnten zu einem öffentlichen Ort der Übersetzung und Vermittlung werden, nicht nur zwischen unterschiedlich befähigten Subjekten, sondern auch zwischen verschiedenen, oft widersprüchlichen Interessen und Bedürfnissen, die eine gemeinsame Artikulation und Organisation von sozialen Kämpfen innerhalb von Sorgeverhältnissen erschweren. Neben der drängenden Frage nach der Umsetzung einer geschlechter- und sozial gerechten Arbeitsverteilung betrifft dies beispielsweise auch den Wunsch von Menschen mit Beeinträchtigungen, nicht als ,Pflegefall' betrachtet zu werden und den eigenen Unterstützungsbedarf vorzugsweise über vertraute Personen oder nicht-professionelle Assistent_innen abzudecken; ein Bedürfnis, das gegenwärtig im Konflikt mit den Interessen von Pflegekräften steht, die für die Wertschätzung ihrer Professionalität in Zeiten der De-Professionalisierung von Pflege kämpfen.

Die ,demenzfreundliche Kommune ließe sich hier perspektivisch als heterogener, durchlässiger Raum, als „common space“ und Gemeingut im Sinne von Stavrides (2016) vorstellen. Sie könnte zu einem öffentlichen Ort werden, an dem sich Menschen ausgehend von ihren alltäglichen und nicht selten konfligierenden Sorgen und Bedürfnissen treffen, um die Frage, wie eine sozial gerechte und lebbare Ausgestaltung der Daseinsvorsorge aussehen müsste, zu einer gemeinsamen Frage zu machen. Dies ist eine Voraussetzung für politische Organisation und stellt die Grundlage dafür dar, zu einem der Labore für neuartige soziale Beziehungen und Sorgepraktiken zu werden, die über die Gegenwart hinausreichen: um „uns selbst und unseren geteilten Alltag zu verändern - hin zu einer in der Tat emanzipierten Gesellschaft“ (ebd.: 58).

\section{Endnoten}

[1] Mein Dank gilt den anonymen Gutachter_innen und der s u b / u r b a n-Redaktion, insbesondere Nina Schuster und Stefan Höhne, deren konstruktive Überarbeitungsvorschläge dem vorliegenden Aufsatz sehr zugute kamen. Für ihre Unterstützung möchte ich zudem Alina Gothe herzlich danken, die als studentische Mitarbeiterin in dem von mir geleiteten Forschungsprojekt „Die Vergesellschaftung der Demenz“ arbeitet. 
[2] Der Begriff der so genannten Demenz (lat.: ohne Verstand, geistlos) ist historisch negativ belastet und begünstigt Formen der Stigmatisierung, Viktimisierung und Ausgrenzung von als ,dement‘ oder ,demenzkrank ${ }^{\star}$ kategorisierten Personen. Im Sinne eines reflektierten Gebrauchs der Demenzkategorie werden im Artikel daher alternative Bezeichnungen wie ,Menschen mit Demenzdiagnose“ oder ,Personen mit kognitiven Veränderungen“ bzw. ,Beeinträchtigungen‘ verwendet (vgl. Wißmann 2016: 34f.). Damit sollen zugleich kulturelle und soziale Konstruktionsanteile an der medizinischen Diagnosekategorie markiert werden, ohne die Materialität und leibliche Erfahrung kognitiver Veränderungen in Abrede zu stellen.

[3] Die Einführung der Pflegeversicherung verdeutlicht daher, dass der gegenwärtige Wandel des Wohlfahrtsstaats weniger als Abbau, sondern als Umbau zu verstehen ist, der eine Neujustierung der Beziehungen, Verantwortlichkeiten und Leistungen von Individuum, Gemeinschaft und Staat betreibt (Lessenich 2008). Gerade im Falle der Demenz zeigt sich dies besonders deutlich, da die Leistungsbezüge für Menschen mit Demenzdiagnose in den letzten Jahren eben nicht gekürzt, sondern seit der Einführung eines neuen Pflegebedürftigkeitsbegriffs weiter ausgeweitet wurden (Wegweiser Demenz o. J.). Im Vordergrund stehen hierbei Leistungen zur Inanspruchnahme ambulanter Pflegedienste und niedrigschwelliger Betreuungsdienste, welche vermehrt von ehrenamtlichen, nicht-professionellen ,Pflegehelfer_innen' erbracht werden. Mit der zur Entlastung der pflegenden Angehörigen eingeführten Verhinderungspflege stehen seit 2017 zudem Gelder zur Aufwandsentschädigung einspringender Verwandter, Nachbar_ innen oder Freund_innen zur Verfügung. Komplementär dazu schafft die ,Engagementstrategie“ der Bundesregierung seit 2016 verbesserte Rahmenbedingungen für „eine neue strategische Ausrichtung der Engagementpolitik auf Bundesebene“, die auch auf die aktive Mobilisierung bürgerschaftlichen Engagements im Bereich Demenz zielen (Zwischenbericht Allianz für Demenz 2016: 27).

[4] Das dahinterliegende kapitalismustheoretische Argument schließt an feministischmaterialistische Ansätze an, die seit den 1970er Jahren (u. a. durch eine Re-Lektüre von Rosa Luxemburgs Weiterentwicklung des Konzepts der ,ursprünglichen Akkumulation“ von Marx) eine Kritik an der umfassenden Subsumption der gesellschaftlichen Sorgeverhältnisse unter den kapitalistischen Reproduktionsprozess formulieren (u. a. Federici 2012). Danach sei die Kapitalverwertung auf die kostenlose Aneignung von nicht kapitalisierbaren, sozusagen systemfremden Ressourcen angewiesen, wozu neben natürlichen Ressourcen auch nicht-entlohnte Sorgearbeit zähle, die der Reproduktion der (ehemaligen) Arbeitskraft diene (van Dyk 2017, Haubner 2017).

[5] Das Förderprogramm erstreckte sich über neun Jahre (2008-2015); eine Liste mit den bundesweit geförderten Projekten findet sich bei Rothe, Kreutzner und Gronemeyer (2015: 279-285).

[6] Aus einer Citizenship-Perspektive handelt es sich z.B. um eine Form von sozialrechtlicher Diskriminierung, wenn Demenz lediglich im Rahmen der Sozialen Pflegeversicherung (SGB XI), nicht aber in anderen Sozialgesetzbüchern verortet wird (Wißmann 2016: 20f.). Insbesondere die im SGB IX verankerte, Teilhabe und Rehabilitation von Menschen mit Behinderung،, die u. a. Ansprüche auf Leistungen wie Assistenz und Teilhabehilfe regelt, bleibt Menschen mit kognitiven Veränderungen vorenthalten. Die den kommunalen Verwaltungen übertragene Umsetzung der auch von Deutschland ratifizierten UN-Behindertenrechtskonvention wird dadurch faktisch unterlaufen.

[7] Hier ist allerdings hervorzuheben, dass die Kritik der polit-ökonomischen und wohlfahrtsstaatstheoretischen Rahmenbedingungen hierfür die aktuellen gesellschaftlichen Kräfteverhältnisse verantwortlich macht; der Zusammenhang zwischen einem Ausbau zivilgesellschaftlichen Engagements bei gleichzeitigem Rückbau staatlicher Dienstleistungen wird damit historisch und sozio-politisch kontextualisiert. Demgegenüber finden sich in den gemeinschaftsaffirmierenden Ansätzen im diskursiven Umfeld der „demenzfreundlichen Kommunen“ häufig metaphysische Dualismen, die den per se kalten, rationalen und inhumanen Dienstleistungen der öffentlichen und privaten Daseinsvorsorge die Empathie, Wärme und normative Überlegenheit der sorgenden Gemeinschaft gegenüberstellt, so besonders deutlich bei Gronemeyer (2015). 


\section{Autor_innen}

Mike Laufenberg ist Soziologe und arbeitet $\mathrm{u}$. a. in den Bereichen Geschlechtersoziologie und Queer Theory, Wohlfahrtsstaats- und Arbeitsforschung sowie zu sozialen Ungleichheiten in Organisationen.

mike.laufenberg@tu-berlin.de

Die Publikation dieses Beitrags wurde durch die Deutsche Forschungsgemeinschaft (DFG) ermöglicht.

\section{Literatur}

Aktion Demenz e.V. (o.J.): Willkommen! http://www.demenzfreundliche-kommunen.de (letzter Zugriff am 27.12.2017).

Aktion Demenz e.V. (2009): Demenz und Kommune. Ein praktischer Vorschlag für lokale Aktivitäten. https://www.aktion-demenz.de/images/stories/pdf/aktion_demenzo8.pdf (letzter Zugriff 21.4.2018).

Aselmeier, Laurenz (2007): Community Care und Menschen mit Behinderung. Gemeinwesenorientierte Unterstützung in England, Schweden und Deutschland. Wiesbaden: VS Verlag.

Aust, Andreas / Klenke, Olaf / Mohr, Katrin / Zimmermann, Sabine (2017): Gute soziale Dienstleistungen und Infrastruktur für eine bessere Gesellschaft. In: Barbara Fried / Hannah Schurian (Hg.): Umcare. Gesundheit und Pflege neu organisieren. Berlin: Rosa-Luxemburg-Stiftung, 6-14.

Bartlett, Ruth / O'Connor, Deoborah (2010): Broadening the Dementia Debate. Toward Social Citizenship. Bristol: Policy Press.

Brannelly, Tula (2016): Citizenship and people living with dementia. A case for the ethics of care. In: Dementia 15/3, 304-314.

Brömme, Norbert (1999): Eine neue Kultur des Helfens und der mitmenschlichen Zuwendung? Über die sozialen Auswirkungen des Pflegeversicherungsgesetzes. Veröffentlichungsreihe des Instituts für Pflegewissenschaft an der Universität Bielefeld (IPW). http://www.unibielefeld.de/gesundhw/ag6/downloads/ipw-106.pdf (letzter Zugriff am 26.3.2018).

Bundesministerium des Inneren (o.J.): Jedes Alter zählt. Weiterentwicklung der Demografiestrategie der Bundesregierung. http://www.demografie-portal.de/ DE/Informieren/Dialogprozess/Weiterentwicklung_Demografiestrategie.pdf? blob=publicationFile\&v=12 (letzter Zugriff am 27.12.2017).

Bundesministerium für Familie, Senioren, Frauen und Jugend (BMFSFJ) (2002): Vierter Bericht zur Lage der älteren Generation in der Bundesrepublik Deutschland. Risiken, Lebensqualität und Versorgung Hochaltriger unter besonderer Berücksichtigung demenzieller Erkrankungen. Berlin.

Bundesministerium für Familie, Senioren, Frauen und Jugend (BMFSFJ) (2012): Achter Familienbericht. Zeit für Familie - Familienzeitpolitik als Chance einer nachhaltigen Familienpolitik. https://www.bmfsfj.de/blob/76278/b8a3571fob33egd4152d410c1a7d b6ee/achter-familienbericht-data.pdf (letzter Zugriff 21.4.2018).

Bundesministerium für Familie, Senioren, Frauen und Jugend (BMFSFJ) / Bundesministerium für Gesundheit (Hg.) (2014): Gemeinsam für Menschen mit Demenz. Die Handlungsfelder. https://www.bmfsfj.de/blob/77342/2251ffd429744887fc8528a75 deoff6c/2014-09-15-agenda-allianz-fuer-demenz-data.pdf (letzter Zugriff am 28.3.2018).

Demenz Support Stuttgart (Hg.) (2013): „Ich spreche für mich selbst.” Menschen mit Demenz melden sich zu Wort. Frankfurt am Main: Mabuse.

Demenz Support Stuttgart (Hg.) (2017): Beteiligtsein von Menschen mit Demenz. Praxisbeispiele und Impulse. Frankfurt am Main.: Mabuse.

Enquete-Kommission „Zukunft des bürgerschaftlichen Engagements“ Deutscher Bundestag (Hg.) (2003): Bürgerschaftliches Engagement und Sozialstaat. http://dipbt.bundestag. $\mathrm{de} / \mathrm{doc} / \mathrm{btd} / 14 / 089 / 1408900 . p d f$ (letzter Zugriff 21.4.2018).

Federici, Silvia (2012): Aufstand aus der Küche. Reproduktionsarbeit im globalen Kapitalismus und die unvollendete feministische Revolution. Münster: edition assemblage. 
Gerlinger, Thomas / Röber, Michaela (2012): DieZieleundWirkungender Pflegeversicherung. In: Bundeszentrale für politische Bildung. http://www.bpb.de/politik/innenpolitik/ gesundheitspolitik/72804/ziele-und-wirkungen-der-pflegeversicherung?p=all (letzter Zugriff am 27.3.2018).

Graefe, Stefanie (2010): Autonomie und Teilhabe. Eckpunkte emanzipatorischer Altersforschung. In: Susanne Kümpers / Josefine Heusinger (Hg.): Autonomie trotz Armut und Pflegebedarf? Altern unter Bedingungen von Marginalisierung. Bern: Huber, 249-260.

Gronemeyer, Reimer (2011): Leben mit Demenz - Ein Überblick zur Situation in Deutschland oder Warum die Verwirrtheit ein Schlüssel zum Verständnis unserer verstörenden Gegenwart ist. In: Transferplus 5, 4-8.

Gronemeyer, Reimer (2015): Die Demenzfreundliche Kommune - das Wagnis. In: Verena Rothe / Gabriele Kreutzner / Reimer Gronemeyer (Hg.): Im Leben bleiben. Unterwegs zu demenzfreundlichen Kommunen. Bielefeld: transcript, 17-40.

Haubner, Tine (2017): Die Ausbeutung der sorgenden Gemeinschaft. Laienpflege in Deutschland. Frankfurt am Main/New York: Campus.

Innes, Anthea (2009): Dementia Studies. A Social Science Perspective. London: Sage.

Jones, Ian / Higgs, Paul (2010): The natural, the Normal and the normative. Contested terrains in ageing and old age. In: Social Science \& Medicine 71, 1513-1519.

Katz, Stephen / Westphal, Barbara L. (2004): Is the functional ,normal'? Age, sex and the Biomarking of Successful Living. In: History of the Human Sciences 17/1, 53-75.

Kellehear, Allan (2005): Compassionate Cities. Public Health and End-of-Life Care. New York/Oxford: Routledge.

Kirchen-Peters, Sabine / Hielscher, Volker (2012): Expertise „Nationale Demenzstrategien“. http://www.iso-institut.de/download/Nationale_Demenzstrategien_Endbericht_ BMFSFJ.pdf (letzter Zugriff am 27.12.2017).

Kirchen-Peters, Sabine / Hielscher, Volker (2013): Nationale Demenzstrategien. Vorbilder für Deutschland? In: Informationsdienst Altersfragen 40/2, 18-24.

Klie, Thomas (2014): Wen kümmern die Alten? Auf dem Weg in eine sorgende Gesellschaft. München: Pattloch.

Knijn, Trudi / Kremer, Monique (1997): Gender and the caring dimension of welfare states. Toward inclusive citizenship. In: Social Politics 4/3, 328-361.

Kontos, Pia (2012): Rethinking sociability in long-term care. An embodied dimension of selfhood. In: Dementia 11/3: 329-46.

Kontos, Pia / Miller, Karen-Lee / Kontos, Alexis (2017): Relational citizenship. Supporting embodied selfhood and relationality in dementia care. In: Sociology of Health \& Illness 39/2, 182-198.

Kreutzner, Gabriele (2015): Zwischen Reflexion und konkretem Tun. In: Verena Rothe / Gabriele Kreutzner / Reimer Gronemeyer (Hg.): Im Leben bleiben. Unterwegs zu demenzfreundlichen Kommunen. Bielefeld: transcript, 237-278.

Lane, Heather Patricia / McLachlan, Sue Anne / Philip, Jennifer (2013): The war against dementia: Are we battle weary yet? In: Age and Ageing 42/3, 281-283.

Laufenberg, Mike (2014): Sexualität und Biomacht. Vom Sicherheitsdispositiv zur Politik der Sorge. Bielefeld: transcript.

Leicht, Hanna / König, Hans-Helmut (2012): Krankheitskosten bei Demenz aus gesellschaftlicher Perspektive. Eine Übersicht. In: Bundesgesundheitsblatt 55/5, 677-684.

Lessenich, Stephan (2008): Die Neuerfindung des Sozialen. Der Sozialstaat im flexiblen Kapitalismus. Bielefeld: transcript.

Lokale Allianz für Demenz (o.J.): Lokale Allianzen unterstützen die Allianz für Demenz. https://www.lokale-allianzen.de/programm.html (letzter Zugriff 21.4.2018)

Lutz, Ronald (Hg.) (2012): Erschöpfte Familien. Wiesbaden: Springer VS.

Maier, Maja (2008): Familien, Freundschaften, Netzwerke. Zur Zukunft persönlicher Unterstützungsbeziehungen. In: Sylvia Buchen / Maja Maier (Hg.): Älterwerden neu denken. Interdisziplinäre Perspektiven auf den demografischen Wandel. Wiesbaden: Springer VS, 219-235.

Pflegereport (2017): Gutes Leben mit Demenz: Daten, Erfahrungen und Praxis. Heidelberg: medhochzwei.

Precarias a la deriva (2011): „Was ist dein Streik“. Militante Streifzüge durch die Kreisläufe der Prekarität. Wien/Berlin: Turia+Kant.

Priestley, Mark (1999): Disability Politics and Community Care. London: J. Kingsley. 
Proctor, Gillian (2001): Listening to older women with dementia. Relationships, voices and power. In: Disability \& Society 16/3, 361-376.

Robertson, Ann (1990): The policies of Alzheimer's disease. A case study in apocalyptic demography. In: International Journal of Health Services 20/3, 429-442.

Rodger, John J. (2000): From a Welfare State to a Welfare Society. The Changing Context of Social Policy in a Postmodern Era. Basingstoke: Palgrave Macmillan.

Rohra, Helga (2012): Aus dem Schatten treten. Warum ich mich für unsere Rechte als Demenzbetroffene einsetze. Frankfurt am Main: Mabuse.

Rose, Nikolas (2000): Tod des Sozialen? Eine Neubestimmung der Grenzen des Regierens. In: Ulrich Bröckling / Susanne Krasmann / Thomas Lemke (Hg.): Gouvernementalität der Gegenwart. Studien zur Ökonomisierung des Sozialen. Frankfurt am Main: Suhrkamp, 72-109.

Rothe, Verena (2015): Menschen mit Demenz in der Kommune - das Programm. In: Verena Rothe / Gabriele Kreutzner / Reimer Gronemeyer (Hg.): Im Leben bleiben. Unterwegs zu demenzfreundlichen Kommunen. Bielefeld: transcript, 43-235.

Rothe, Verena / Kreutzner, Gabriele / Gronemeyer, Reimer (Hg.) (2015): Im Leben bleiben. Unterwegs zu demenzfreundlichen Kommunen. Bielefeld: transcript.

Sevenhuijsen, Selma (1998): Citizenship and the Ethics of Care. Feminist Considerations on Justice, Morality, and Politics. New York/Oxford: Routledge.

Siebte Altenberichtskommission (2016): Sorge und Mitverantwortung in der Kommune - Aufbau und Sicherung zukunftsfähiger Gemeinschaften. Und Stellungnahme der Bundesregierung. https://www.siebter-altenbericht.de/index.php?eID=tx_securedown loads $\& \mathrm{p}=1 \& \mathrm{u}=\mathrm{o} \& \mathrm{~g}=\mathrm{o} \& \mathrm{t}=1524408180 \& \mathrm{hash}=\mathrm{cc} 35$ foe8coa999eb60042d 3 eb595bdbf 32 24e976\&file=/fileadmin/altenbericht/pdf/Der_Siebte_Altenbericht.pdf (letzter Zugriff 21.4.2018).

Statistische Ämter des Bundes und der Länder (2010): Demografischer Wandel in Deutschland, Heft 2: Auswirkungen auf Krankenhausbehandlungen und Pflegebedürftige in Bund und Ländern. https://www.destatis.de/DE/Publikationen/Thematisch/ Bevoelkerung/DemografischerWandel/KrankenhausbehandlungPflegebeduer ftige5871102109004.pdf?_blob=publicationFile (letzter Zugriff 21.4.2018).

Stavrides, Stavros (2016): Common Space. The City as Commons. Chicago: University of Chicago Press.

Störmer, Norbert (2002): Community Care - Ende für Großeinrichtungen. Die gemeindepsychiatrische Modernisierung. In: Die Orientierung 25/1, 27-30.

Theobald, Hildegard (2008): Care-Politiken, Care-Arbeitsmarkt und Ungleichheit. Schweden, Deutschland und Italien im Vergleich. In: Berliner Journal für Soziologie 18/2, 257-281.

Tronto, Joan (2010): Creating caring institutions. Politics, plurality, and purpose. In: Ethics and Social Welfare 4/2, 158-171.

Tronto, Joan (2013): Caring Democracy. Markets, Equality and Justice. New York: New York University Press.

van Dyk, Silke (2016): Vorwärts in die Vergangenheit: Postwachstum als Gemeinschaftsprojekt? In: AK Postwachstum (Hg.): Wachstum - Krise und Kritik. Frankfurt am Main: Campus, 245-268.

van Dyk, Silke (2017): Community-Kapitalismus oder Alternativökonomie? Kritische Anmerkungen zur Wiederentdeckung des Gemeinsinns. In: Geschlossene Gesellschaften. Verhandlungen des 38. Kongresses der Deutschen Gesellschaft für Soziologie in Bamberg 2016. http://publikationen.soziologie.de/index.php/kongressband_2016/article/ view/648/pdf (letzter Zugriff 21.4.2018).

van Dyk, Silke / Dowling, Emma / Haubner, Tine (2016): Für ein rebellisches Engagement. In: Blätter für deutsche und internationale Politik 2/2016, 37-40.

Wegleitner, Klaus / Heimerl, Katharina / Kellehear, Allan (2015): Compassionate Communities. Case Studies from Britain and Europe. New York/Oxford: Routledge.

Wegweiser Demenz (o.J.): Pflegeversicherung - Leistungen im Überblick. https://www. wegweiser-demenz.de/informationen/gesetzliche-leistungen/pflegeversicherung.html (letzter Zugriff am 28.3.2018).

Wetzstein, Verena (2010): Alzheimer-Demenz. Perspektiven einer integrativen Demenz-Ethik. In: Markus Christen / Corinna Osman / Ruth Baumann-Hölzle (Hg.): Herausforderung Demenz. Spannungsfelder und Dilemmata in der Betreuung. Bern: Peter Lang, 53-70. 
Wißmann, Peter (2016): Demenz: Ausschluss aus der inklusiven Gesellschaft? Berlin: Verlag des Deutschen Vereins für öffentliche und private Fürsorge e.V.

Wißmann, Peter (2017a): Beteiligt werden, beteiligt sein, beteiligt bleiben. Ein Problemaufriss. In: Demenz Support Stuttgart (Hg.): Beteiligtsein von Menschen mit Demenz. Praxisbeispiele und Impulse. Frankfurt am Main.: Mabuse, 17-35.

Wißmann, Peter (2017b): Vor der Haustüre spielt die Musik. Sich in Planungsprozesse vor Ort einbringen. In: Demenz Support Stuttgart (Hg.): Beteiligtsein von Menschen mit Demenz. Praxisbeispiele und Impulse. Frankfurt am Main.: Mabuse, 121-126.

Wißmann, Peter / Gronemeyer, Reimer (2008): Demenz und Zivilgesellschaft. Eine Streitschrift. Frankfurt am Main: Mabuse.

Zwischenbericht Allianz für Demenz (2016): Zwischenbericht September 2016 zur Umsetzung der Agenda. Gemeinsam für Menschen mit Demenz. https://www.allianzfuer-demenz.de/fileadmin/de.allianz-fuer-demenz/content.de/bilder/meldungen/1609-21_Zwischenbericht/Zwischenbericht_Gemeinsam_fuer_Menschen_mit_Demenz_ barrierefrei.pdf (letzter Zugriff 21.4.2018).

\section{Caring Communities? "Dementia friendly" communes between lean social welfare model and emancipation}

The author analyzes the "dementia friendly" reorganization of society as proclaimed by the German federation, local communes and self-help organizations. He reconstructs the political-economic and welfare state conditions of the policy concept of "caring communities" which declares the civic society a crucial player within the "dementia friendly" city. Under current conditions this development runs the risk of compensating structural gaps within the publicly funded system of elderly care. However, by further exploring the civil rights concept of "dementia-friendly communities" it is stated that communal and civic forms of collective caring can simultaneously help to enforce emancipatory forms of participation and self-determination for people with dementia. Against this backdrop the author contours an alternative analytic framing for community-based arrangements of care that avoids the analytical dichotomy of either considering it a lean social welfare model or idealizing it as a warm and caring community. 